\title{
An active tooth surface design methodology for face-hobbed hypoid gears based on measuring coordinates
}

\author{
Du Jinfu, ${ }^{\mathrm{a}, \mathrm{b}, *}$, Fang Zongde ${ }^{\mathrm{b}}$ \\ ${ }^{a}$ School of Mechanical and Precision Instrument Engineering,Xi'an University of Technology,Xi'an 710048, China \\ ${ }^{\mathrm{b}}$ Department of Mechanical Engineering, Northwestern Polytechnical University, Xi'an 710072,China
}

Abstract: An active tooth surface design methodology is proposed for face-hobbed hypoid gears based on fitting the measuring coordinates of real tooth surface that includes the flank deviations caused by machine errors and the deformation of heat treatment. Firstly, non-uniform rational B-spline(NURBS) is used as a tool to fit the discrete measuring coordinates of gear and pinion, then real tooth surface equations are obtained. Secondly, the pinion auxiliary tooth surface that is in line contact with the gear real tooth surface and has a parabolic transmission error(TE) is obtained by using the gear as a virtual cutter to generate the pinion under a predesigned motion function, then the pinion target tooth surface is obtained by modifying the pinion auxiliary tooth surface along the contact line with a predesigned contact pattern(CP). Thirdly, an optimization model is proposed to solve the adjustments of pinion machining parameters, this model is solved by using sequence quadratic program(SQP). The feasibility of this methodology is demonstrated by using a numerical example of a Klingelnberg-Oerlikon's Spirac hypoid gear set, the results

* Corresponding author. Tel.: +86 15991945157.

E-mail addresses: robin367@qq.com (J. Du), fauto@nwpu.edu.cn (Z.Fang). 
are in line with the preconditions. This proposed methodology provides a new approach for meshing performances control of face-hobbed hypoid gears in the phase of trial-manufacture.

Keywords: face-hobbed hypoid gear; active tooth surface design; non-uniform rational B-spline; modification; real tooth surface; Spirac cutting system

\section{Introduction}

Face-hobbed hypoid gears are widely used in the automobile industry to transfer power between two crossed axes. To meet the environmental noise requirements and the customer demands, vibration, noise and working life has been a major concern in the design of automobile gear transmission systems. A significant volume of tooth surface design methods are proposed to control the meshing performances, including the TE and the CP. Litvin et al. [1-3] proposed a local synthesis technique and TCA approach for a predesigned TE curve and contact path orientation that produced lower gear running noise and assembly sensitivity, however, the meshing performances of the region away from the reference point are difficult to control. Fang et al. [4,5] proposed a new approach based on a predesigned parabolic function of TE and a predesigned linear function of contact path, that the meshing performances in whole tooth contact could be controlled directly in design. A predesigned second-order parabolic function of TE has been successfully applied by Gonzalez-Perez [6] to improving the meshing performances of involute helical gears. Simon [7] proposed an 
optimization design method for optimal machine settings based on maximal contact pressure, load distribution factor, and TE of hypoid gears. In addition, Wang and Fong [8] proposed a methodology for synthesizing the tooth surfaces of a face-milled spiral bevel gear set with a predetermined fourth-order motion curve. A manufacturing process for fabricating a cylindrical crown gear drive is provided with a controllable fourth-order polynomial function of TE by Lee [9] to reduce the level of gear running noise and to avoid edge contact. Fang et al. $[10,11]$ proposed a higher-order polynomial function of TE and present the advantages of it over second-order and forth-order polynomial functions of TE in terms of load sharing, bending, tensile and contact stresses. Artoni et al. [12,13] presented a LTCA method that employed a semi-analytical shell instead of a FEM of hypoid gears, and proposed a novel methodology to systematically define optimal ease-off topography to improve the meshing performances of hypoid gear drive. ShiP[14] proposed an ease-off based flank modification methodology both for spiral bevel and hypoid gears made by a modern Cartesian-type hypoid generator, which can serve as a basis for flank modification for other gear types.

The above approaches, however, are based on theoretical tooth surfaces, without considering the tooth surface error caused by the machining errors and the deformation of heat treatment that unavoidable in practical production. Due to the existence of this error, the real tooth surface will deviate from the design surface, which will consequently lead to the failure of full achievement of expecting meshing performances. To make up this defect, flank correction technology is proposed. 
However, here comes up another problem that if the design tooth surface is still used as target surface, both the gear and pinion tooth surface errors must be modified to acquire the predesigned meshing performances. However, in practical production usually only the tooth surface error of the pinion is corrected. Therefore, the negligence of the error of gear real tooth surface from the design surface will result in the deviation of final meshing performances, which will lead to the need of further correction. In the first correction, the real tooth surface of the pinion is close to the target tooth surface, so the second correction is an empirical adjustment according to the $\mathrm{CP}$, which is time-consuming and not precise enough. Kawasaki[15] proposed a new method for remanufacturing large-sized skew bevel gears, and the pinion or gear member that has good performance mating with an existing gear or pinion member can be machined by a CNC machining center.

The aim of this work is to propose an active tooth surface design methodology based on real tooth surfaces for face-hobbed hypoid gears. The first step is to fit the discrete measuring coordinates into complete tooth surface and deduce the real tooth surface equations by using NURBS fitting method. The second step is to apply the gear real surface as a virtual cutter to generate the pinion auxiliary tooth surface according to the predesigned motion function in meshing coordinate system. This auxiliary tooth surface is in line contact with the gear real tooth surface and satisfies the predesigned TE. Then the pinion auxiliary tooth surface is modified along the contact line according to the predesigned modification curve to fulfill point contact. Finally, the least sum of squared errors optimization model is developed, and a weight 
factor is introduced to selectively control the meshing performances of a certain side. Here, SQP is used as a tool to solve the adjustments of pinion machining parameters. Thus, the tooth surface errors of both gear and pinion are all considered by just correcting the pinion surface as in practical production, and no second correction is needed. The feasibility of this active tooth surface design methodology is clearly illustrated by a numerical example that uses a Klingelnberg-Oerlikon's Spirac high speed axle hypoid gear set cut by C28 hypoid generator.

There are three premises for this methodology:

(1) The errors caused by inaccuracy of measuring equipment and misoperation are not considered.

(2) Repeatability exists in the tooth surface errors caused by machining errors and the deformation of heat treatment.

(3) Tooth surface fitting is precise enough.

\section{Distribution of measuring points}

The measurement of tooth surface is carried out by the gear measuring center according to the pre-divided mesh on theoretical tooth surfaces, so the deduction of the theoretical tooth surfaces is prerequisite.

\subsection{Deduce of theoretical tooth surface}

The tooth machining parameters first obtained by the setting parameters tables are called theoretical machining parameters in this work, and the machined tooth 
surface is called theoretical tooth surface. The acquirement of theoretical machining parameter refers to Ref [16]. Take the machining of generated LH pinion as an example. As shown in Fig. $1, S_{R}$ is the radial distance of the cradle, $i$ is the tilted angle, $j$ is the swivel angle of the cutting tilting, $\phi_{1}$ is the rotation angle of the work gear, $\beta$ is the rotation angle of the cutter head, $E_{m}$ is the vertical offset, $\Delta A$ is the increment of machine center to back, $\Delta B$ is the sliding base feed setting, $\gamma_{m}$ is the machine root angle, $q_{1}=\theta_{c}+\phi_{c}$, where $\theta_{c}$ is the initial cradle setting angle, $\phi_{c}=\phi_{c 2}-\phi_{c 1}$ is the sum of cradle rotation angle, $\phi_{c 2}=\phi_{1} / R_{a}$ is the generating cradle rotation angle, $R_{a}$ is the roll ratio, $\phi_{c 1}=\beta z_{0} / z_{p}$ is the epicycloidal cradle rotation angle, $z_{0}$ is the number of cutter starts, $z_{p}$ is the tooth number of the imaginary generating gear. $\mathrm{S}_{1}\left(x_{1}, y_{1}, z_{1}\right)$ and $\mathrm{S}_{t}\left(x_{t}, y_{t}, z_{t}\right)$ are the coordinate systems rigidly connected to the work gear and the cutter head. $S_{a}, S_{b}, S_{c}, S_{d}, S_{e}, S_{f}$ are auxiliary coordinate systems. In this paper, the blade edge function $\boldsymbol{r}_{t}(u)$ represented in coordinate system $\mathrm{S}_{\mathrm{t}}$ is assumed to be given [17].

$$
\boldsymbol{r}_{t}(u)=\boldsymbol{r}_{t}\left(\alpha_{0}, r_{c}, \delta_{0}, r_{0}: u\right)
$$

Where, $u$ is blade parameter, $\alpha_{0}$ is the profile angle, $r_{c}$ is the blade curvature radius, $\delta_{0}$ is the offset angle, $r_{0}$ is the cutter radius.

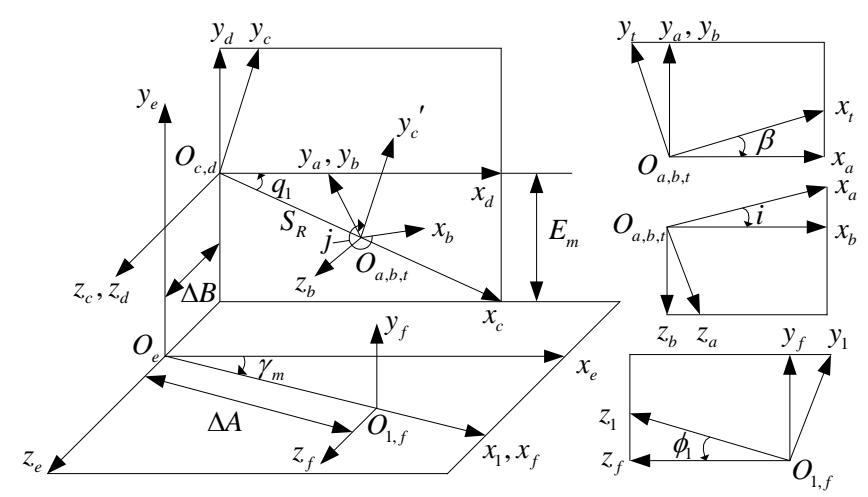

Fig.1 Cutting coordinate systems of the LH pinion 
The position and unit normal vectors of the theoretical pinion tooth surface can then be represented in coordinate system $S_{1}$ as $\boldsymbol{r}_{L 1}$ and $\boldsymbol{n}_{L 1}$ by applying the following homogeneous coordinate transformation matrix equation:

$$
\left\{\begin{array}{l}
\boldsymbol{r}_{L 1}\left(\zeta ; u, \beta, \phi_{1}\right)=\boldsymbol{M}_{1 t} \boldsymbol{r}_{t}(u) \\
\boldsymbol{n}_{L 1}\left(\zeta ; u, \beta, \phi_{1}\right)=\frac{\frac{\partial \boldsymbol{r}_{L 1}}{\partial_{u}} \times \frac{\partial \boldsymbol{r}_{L 1}}{\partial_{\beta}}}{\left|\frac{\partial \boldsymbol{r}_{L 1}}{\partial_{u}} \times \frac{\partial \boldsymbol{r}_{L 1}}{\partial_{\beta}}\right|}
\end{array}\right.
$$

where

$$
\begin{aligned}
& \boldsymbol{M}_{1 t}=\boldsymbol{M}_{1 f}\left(\phi_{1}\right) \boldsymbol{M}_{f a}(\boldsymbol{\zeta}) \boldsymbol{M}_{a t}(\beta)=\left[\begin{array}{llll}
c_{11} & c_{12} & c_{13} & c_{14} \\
c_{21} & c_{22} & c_{23} & c_{24} \\
c_{31} & c_{32} & c_{33} & c_{34} \\
c_{41} & c_{41} & c_{43} & c_{44}
\end{array}\right], \\
& c_{11}=\cos \gamma_{m}\left[\sin \left(\theta_{c}+\phi_{c}-j\right) \cos i \cos \beta-\cos \left(\theta_{c}+\phi_{c}-j\right) \sin \beta\right]-\sin \gamma_{m} \sin i \cos \beta, \\
& c_{12}=\cos \gamma_{m}\left[-\sin \left(\theta_{c}+\phi_{c}-j\right) \cos i \sin \beta-\cos \left(\theta_{c}+\phi_{c}-j\right) \cos \beta\right]-\sin \gamma_{m} \sin i \sin \beta, \\
& c_{13}=\cos \gamma_{m} \sin i \sin \left(\theta_{c}+\phi_{c}-j\right)+\sin \gamma_{m} \cos i, \\
& c_{14}=S_{R} \cos \gamma_{m} \cos \left(\theta_{c}+\phi_{c}\right)-\Delta A-\sin \gamma_{m} \Delta B, \\
& c_{21}=\sin \phi_{1} \sin \gamma_{m}\left[\sin \left(\theta_{c}+\phi_{c}-j\right) \cos i \cos \beta-\cos \left(\theta_{c}+\phi_{c}-j\right) \sin \beta\right]+\cos \phi_{1}\left[\operatorname { c o s } \left(\theta_{c}+\right.\right. \\
&\left.\left.\phi_{c}-j\right) \cos i \cos \beta+\sin \left(\theta_{c}+\phi_{c}-j\right) \sin \beta\right]+\sin \phi_{1} \cos \gamma_{m} \sin i \cos \beta, \\
& c_{22}=\sin \phi_{1} \sin \gamma_{m}\left[-\sin \left(\theta_{c}+\phi_{c}-j\right) \cos i \sin \beta-\cos \left(\theta_{c}+\phi_{c}-j\right) \cos \beta\right]+\cos \phi_{1}\left[-\cos \left(\theta_{c}+\right.\right. \\
&\left.\left.\phi_{c}-j\right) \cos i \sin \beta+\sin \left(\theta_{c}+\phi_{c}-j\right) \cos \beta\right]-\sin \phi_{1} \cos \gamma_{m} \sin i \sin \beta, \\
& c_{23}=\sin \phi_{1} \sin \gamma_{m} \sin i \sin \left(\theta_{c}+\phi_{c}-j\right)+\cos \phi_{1} \sin i \cos \left(\theta_{c}+\phi_{c}-j\right)-\sin \phi_{1} \cos \gamma_{m} \cos i, \\
& c_{24}= S_{R} \sin \phi_{1} \sin \gamma_{m} \cos \left(\theta_{c}+\phi_{c}\right)-S_{R} \cos \phi_{1} \sin \left(\theta_{c}+\phi_{c}\right)+E_{m} \cos \phi_{1}+\Delta B \sin \phi_{1} \cos \gamma_{m} \\
& c_{31}= \cos \phi_{1} \sin \gamma_{m}\left[\sin \left(\theta_{c}+\phi_{c}-j\right) \cos i \cos \beta-\cos \left(\theta_{c}+\phi_{c}-j\right) \sin \beta\right]+\sin \phi_{1}\left[\operatorname { c o s } \left(\theta_{c}+\right.\right. \\
&\left.\left.\phi_{c}-j\right) \cos i \cos \beta+\sin \left(\theta_{c}+\phi_{c}-j\right) \sin \beta\right]-\cos \phi_{1} \cos \gamma_{m} \sin i \cos \beta, \\
&\left.\left.\phi_{c}-j\right) \cos i \sin \beta+\sin \left(\theta_{c}+\phi_{c}-j\right) \cos \beta\right]+\cos \phi_{1} \cos \gamma_{m} \sin i \sin \beta, \\
& \sin \gamma_{m}\left[-\sin \left(\theta_{c}+\phi_{c}-j\right) \cos i \sin \beta-\cos \left(\theta_{c}+\phi_{c}-j\right) \cos \beta\right]+\sin \phi_{1}\left[-\cos \left(\theta_{c}+\right.\right.
\end{aligned}
$$




$$
\begin{aligned}
& c_{33}=\cos \phi_{1} \sin \gamma_{m} \sin i \sin \left(\theta_{c}+\phi_{c}-j\right)+\sin \phi_{1} \sin i \cos \left(\theta_{c}+\phi_{c}-j\right)+\cos \phi_{1} \cos \gamma_{m} \cos i, \\
& c_{34}=S_{R} \cos \phi_{1} \sin \gamma_{m} \cos \left(\theta_{c}+\phi_{c}\right)-S_{R} \sin \phi_{1} \sin \left(\theta_{c}+\phi_{c}\right)+E_{m} \sin \phi_{1}-\Delta B \cos \phi_{1} \cos \gamma_{m}, \\
& c_{41}=c_{42}=c_{43}=0, c_{44}=1 \\
& \zeta=\left(i, j, \theta_{c}, S_{R}, E_{m}, \Delta A, \Delta B, \gamma_{m}, R_{a}, \alpha_{0}, r_{c}, \delta_{0}, r_{0}\right)
\end{aligned}
$$

Equations (1) to (3) can be used to solve the nongenerated theoretical tooth surfaces, the solution for generated ones, however, requires the following meshing equation:

$$
f_{1 t}\left(u, \beta, \phi_{1}\right)=\boldsymbol{n}_{L 1} \boldsymbol{v}_{1}^{\left({ }^{(t)}\right.}=\boldsymbol{n}_{L 1}\left(\dot{\phi}_{1} \frac{\partial \boldsymbol{r}_{L 1}}{\partial \phi_{1}}\right)=0
$$

Where, $\boldsymbol{v}_{1}^{(t)}$ is the relative velocity between the blade edge and the pinion surface represented in coordinate system $\mathrm{S}_{1}$.

Similarly, the matched RH gear theoretical tooth surface can be represented as follows:

$$
\boldsymbol{r}_{L 2}\left(u, \beta, \phi_{2}\right)=\boldsymbol{M}_{2 f}\left(\phi_{2}\right) \boldsymbol{M}_{f t}(\beta) \boldsymbol{r}_{t}(u)
$$

\subsection{Mesh dividing of tooth surface}

During the measurement of tooth surface, the density of measuring points will directly affect subsequent fitting accuracy of the tooth surface. The denser the points distribute, the more accurate the fitting will be, but is time-consuming, which will affect the efficiency. In this paper, a P65 gear measuring center is used, the number of chosen measure points is 225 , that is 15 along the profile and 15 along the tooth trace. As shown in Fig.2, $s$ and $f$ are surface parameters along the directions of the profile and the tooth trace. 


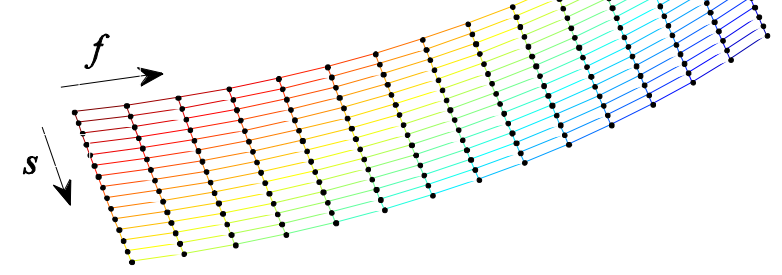

Fig. 2 Distribution of measuring points

\section{Fitting of real tooth surface}

3.1 Errors caused by the machining errors and the deformation of heat treatment

It is well known that the tooth surface errors caused by the machining errors and the deformation of heat treatment are unavoidable in practical production. But they are considered to be repeatable if the following conditions are met: the production batch, the blank material, the machine tool and machine process, the heat treating equipment and technology, the measuring equipment are all the same. This repeatability can be obtained by analyzing the measuring results in the phase of trial-manufacture.

For accuracy and without loss of generality, the average error of each measuring point is calculated based on the measuring results, and the discrete coordinates of the real tooth surface of gear and pinion $r_{M p i j}^{\prime}$ are obtained:

$$
\boldsymbol{r}_{M p i j}^{\prime}=\frac{1}{n} \sum_{k=1}^{n}\left(\boldsymbol{r}_{M p i j}^{(k)}-\boldsymbol{r}_{L p i j}^{(k)}\right) \cdot \boldsymbol{n}_{L p i j}^{(k)}
$$

Where, $p=1,2$ represents the pinion and the gear, $n$ represents the number of measured tooth surfaces, $i, j$ represent the row and column number of the measuring 
point, $\boldsymbol{r}_{M}^{(k)}$ represents the measuring results of surface $k$.

\subsection{Tooth surface fitting}

The discrete tooth surface coordinates must be fitted into complete tooth surface and the real tooth surface equation must be obtained, which is the prerequisite to carry out the subsequent tooth design and contact analysis. NURBS is used here as a tool.

The function of second-order continuous bicubic NURBS surface is represented by an equation $R(s, f)$ as follows [18]:

$$
R(s, f)=\frac{\sum_{i=1}^{m} \sum_{j=1}^{n} N_{i, 3}(s) N_{j, 3}(f) W_{i, j} P_{i, j}}{\sum_{i=1}^{m} \sum_{j=1}^{n} N_{i, 3}(s) N_{j, 3}(f) W_{i, j}}
$$

Where, $m$ and $n$ represent the number of control vertices along the directions of $s$ and $f . P_{i, j}$ represents the control vertices of surface, $W_{i, j}$ is the weight factor of $P_{i, j}, \quad N_{i, 3}$ and $N_{j, 3}$ represent the cubic B-spline primary functions for $s$ and $f$ directions.

Firstly, the control vertices in various NURBS curves are obtained by calculating the measuring data towards the direction of $s$ (or $f$ ), and then all the control vertices in the NURBS surface can be obtained by using the above control vertices as new input points to calculate towards the direction of $f$ (or $s$ ). After that the bicubic NURBS surface can be obtained by putting all the control vertices in to equation (7). The measuring data obtained from the gear measuring center usually don't contains the boundary points, however, they can be obtained by extrapolating that has little influence on the back-calculated control matrix. Finally, the function of real tooth 
surfaces can be obtained through interpolation and back-calculation:

$$
\left\{\begin{array}{l}
\boldsymbol{r}_{M i}^{\prime}=\boldsymbol{r}_{M i}^{\prime}(s, f) \\
\boldsymbol{n}_{M i}^{\prime}(s, f)=\frac{\frac{\partial \boldsymbol{r}_{M i}^{\prime}}{\partial_{s}} \times \frac{\partial \boldsymbol{r}_{M i}^{\prime}}{\partial_{f}}}{\left|\frac{\partial \boldsymbol{r}_{M i}^{\prime}}{\partial_{s}} \times \frac{\partial \boldsymbol{r}_{M i}^{\prime}}{\partial_{f}}\right|},(i=1,2)
\end{array}\right.
$$

Where, the subscript 1 and 2 represent the pinion and the gear.

Then, the tooth surface design and contact analysis can be carried out. A general tooth surface fitting and real tooth surface TCA program was produced for face-hobbed hypoid gears in an earlier work [19].

\subsection{Verification of fitting accuracy}

Since the tooth surface fitted by NURBS is a structural surface, that means apart from control vertices, the other positions on the surface are all calculated through fitting algorithm. The fitting accuracy of NURBS must be verified, and the verification steps are as follows:

(1) Pick $15 \times 15$ points on the theoretical tooth surface in the same position, and then the fitting tooth surface of the theoretical tooth surface can be obtained by the above fitting method.

(2) Work out all the midpoints $(14 \times 14)$ of the minimum surface patches constructed by the adjacent 4 points on the theoretical tooth surface.

(3) Calculate the distance between the midpoints on the theoretical tooth surface and the corresponding points on the fitted tooth surface, among which the largest distance is defined as the maximum fitting error. 
(4) Pick the largest distance in each column to draw the fitting errors curve.

The fitting accuracy of the numerical example in this paper (see section 5) is shown in Fig.3. The fitting error of the pinion tooth surface is relatively large, and the largest fitting errors for gear and pinion tooth surface are all within $0.1 \mu \mathrm{m}$.

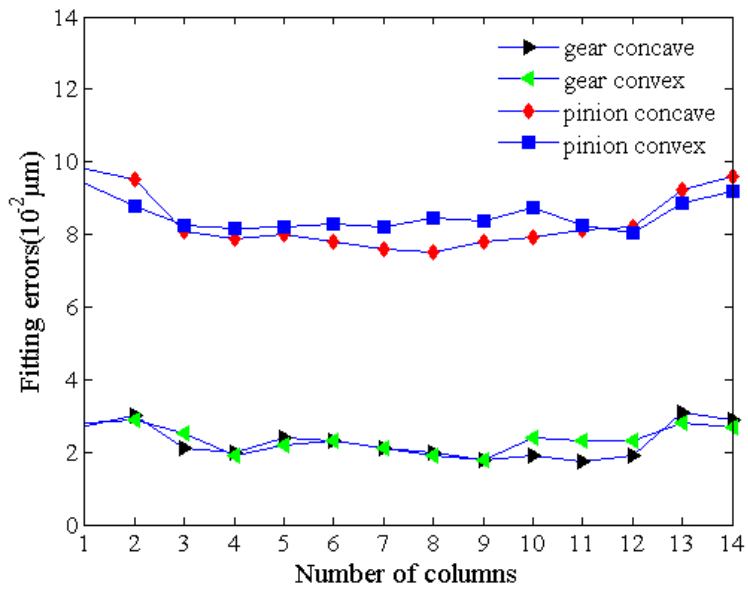

Fig. 3 Curve of fitting errors

\section{Active tooth surface design based on real tooth surfaces}

Active tooth surface design is to design the topography of tooth surface based on the functional requirements. Firstly, the TE curve and the optimal CP are predesigned, and then work out the topography of tooth surface that can precisely satisfy the above predesigned meshing performances and its processing method.

\subsection{Predesigned meshing performances}

\subsubsection{Predesigned TE}

TE has a significant impact on vibration and noise. A volume of researches[1-6] showed that parabolic function of TE can absorb the linear errors caused by installation errors while keep the shape of TE curve. Thus a second-order parabolic 
function of TE is predesigned in this work. As shown in Fig.4, $\varphi_{1}$ is the rotation angle of pinion, $\Delta \varphi_{2}$ is the TE, $\varphi_{1 N}, \varphi_{1 M}, \varphi_{1 P}$ are the pinion rotation angles at the meshing points $N, M$ and $P, \delta_{T E}$ is the maximal TE, $z_{1}$ is the tooth number of pinion, $2 \pi / z_{1}$ represents the pinion rotation angle in a meshing period.

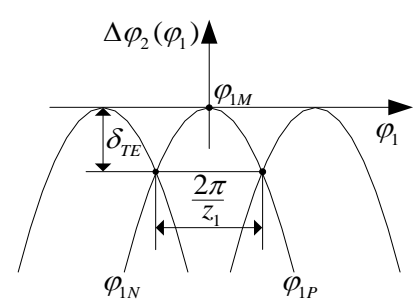

Fig.4 Parabolic transmission error curve

A motion equation can then be obtained according to Fig.4:

$$
\left\{\begin{array}{l}
\varphi_{2}=\varphi_{2}^{(0)}+\frac{z_{1}}{z_{2}}\left(\varphi_{1}-\varphi_{1}^{(0)}\right)+\Delta \varphi_{2}\left(\varphi_{1}\right) \\
\Delta \varphi_{2}\left(\varphi_{1}\right)=-\delta_{T E} \frac{z_{1}^{2}}{\pi^{2}}\left(\varphi_{1}-\varphi_{1}^{(0)}\right)^{2}
\end{array}\right.
$$

Where, $\Delta \varphi_{2}\left(\varphi_{1}\right)$ is the function of TE predesigned, $\varphi_{1}^{(0)}, \varphi_{2}^{(0)}$ are the initial angles of pinion and gear, $z_{2}$ is the tooth number of gear.

\subsubsection{Predesigned CP}

The shape, size and position of the CP directly affect the motion and lubrication performances, transmission efficiency and carrying capacity of the gear pair. The design of the $\mathrm{CP}$ is carried out on the rotary projection of gear real tooth surface, as shown in Fig.5. To reduce the sensitivity to misalignments, the contact path is designed as a straight line [1]. $\eta$ is the predesigned direction angle of the contact path. The major axis of instant contact ellipse is determined by contact area, which is usually designed to be in a middle position, approximately $40 \%-60 \%$ of the tooth 
width direction.

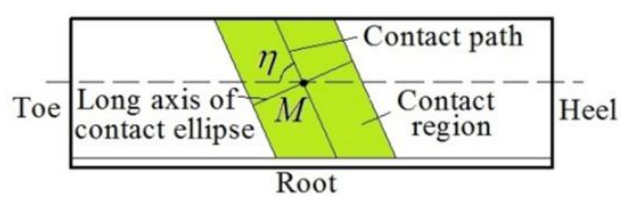

Fig.5 Contact pattern

4.2 Pinion auxiliary tooth surface

The gear real tooth surface that obtained above by fitting is used as a virtual cutter to generate the pinion auxiliary tooth surface under a predesigned motion function in meshing coordinate system, as shown in Fig.6 and Fig.7.

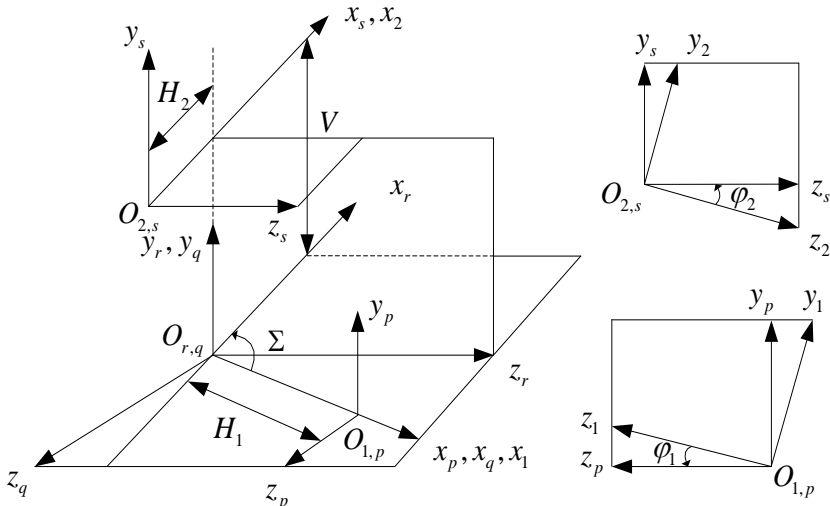

Fig.6 Mesh coordinate systems

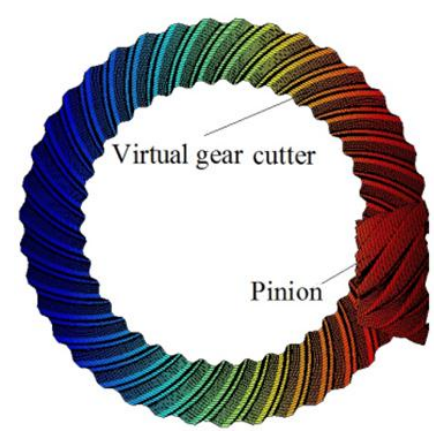

Fig.7 Virtual gear cutter

Where, $H_{1}, H_{2}$ are the axial settings of pinion and gear. $V$ is the offset. $\Sigma$ is the shaft angle. $\mathrm{S}_{2}\left(x_{2}, y_{2}, z_{2}\right)$ is the coordinate system rigidly connected to the gear, $\mathrm{S}_{p}, S_{q}, S_{r}, S_{s}$ are auxiliary coordinate systems.

If the motion function shown in Formula(10) and the meshing function(11) are satisfied in the generating process, the pinion conjugate tooth surface $\Sigma_{g}$ that is completely conjugated with the gear real tooth surface can be obtained:

$$
\varphi_{2}-\varphi_{2}^{(0)}=\frac{z_{1}}{z_{2}}\left(\varphi_{1}-\varphi_{1}^{(0)}\right)
$$




$$
f_{12}\left(u, \beta, \phi_{1}\right)=\boldsymbol{n}_{1} \boldsymbol{v}_{1}^{(21)}=\boldsymbol{n}_{1}\left(\dot{\phi}_{1} \frac{\partial \boldsymbol{r}_{1}}{\partial \phi_{1}}\right)=0
$$

Where, $v_{1}^{(21)}$ is the relative velocity between the surface of gear cutter and the pinion surface represented in coordinate system $\mathrm{S}_{1}$.

For two completely conjugated tooth surfaces there is no TE in every contact position, however, this kind of theoretically perfect tooth surface is totally unadjustable, that means any manufacturing or installation error or bearing deformation will lead to the edge contact and load concentration on tooth surface. Therefore, the matched tooth surfaces are usually designed to be in point contact with a second-order or higher-order parabolic function of TE. Usually, only the pinion tooth surfaces are modified in practical production.

To make the meshing of the gear and pinion satisfy the predesigned TE curve, in the above generating process, Formula(10) is substituted with Formula(9) to alter the original rotation relationship. Then the pinion auxiliary tooth surface $\Sigma_{t}$ is obtained, which is in line contact with the gear real tooth surface, and satisfy the predesigned second-order parabolic function of TE $\Delta \varphi_{2}\left(\varphi_{1}\right)$. The position and unit normal vectors of pinion auxiliary tooth surface are denoted as $\boldsymbol{r}_{A 1}$ and $\boldsymbol{n}_{A 1}$, respectively.

$$
\left\{\begin{aligned}
\boldsymbol{r}_{A 1}\left(s, f, \varphi_{1}\right)= & \boldsymbol{M}_{12} \boldsymbol{r}_{M 2}^{\prime}(s, f) \\
\boldsymbol{n}_{A 1}\left(s, f, \varphi_{1}\right)= & \frac{\frac{\partial \boldsymbol{r}_{M 2}^{\prime}}{\partial_{s}} \times \frac{\partial \boldsymbol{r}_{M 2}^{\prime}}{\partial_{f}}}{\left|\frac{\partial \boldsymbol{r}_{M 2}^{\prime}}{\partial_{s}} \times \frac{\partial \boldsymbol{r}_{M 2}^{\prime}}{\partial_{f}}\right|}
\end{aligned}\right.
$$

Where 


$$
\begin{aligned}
& \boldsymbol{M}_{12}=\boldsymbol{M}_{1 s}\left(V, \Sigma, H_{1}, H_{2}, \varphi_{1}\right) \boldsymbol{M}_{s 2}\left(\varphi_{2}\right)=\left[\begin{array}{llll}
d_{11} & d_{12} & d_{13} & d_{14} \\
d_{21} & d_{22} & d_{23} & d_{24} \\
d_{31} & d_{32} & d_{33} & d_{34} \\
d_{41} & d_{42} & d_{43} & d_{44}
\end{array}\right] \\
& d_{11}=\cos \Sigma, d_{12}=-\sin \varphi_{2} \sin \Sigma, d_{13}=\cos \varphi_{2} \sin \Sigma, d_{14}=-H_{1}-H_{2} \cos \Sigma, \\
& d_{21}=\sin \varphi_{1} \sin \Sigma, d_{22}=\cos \varphi_{1} \cos \varphi_{2}+\cos \Sigma \sin \varphi_{1} \sin \varphi_{2}, \\
& d_{23}=\cos \varphi_{1} \sin \varphi_{2}-\cos \Sigma \cos \varphi_{2} \sin \varphi_{1}, d_{24}=V \cos \varphi_{1}-H_{2} \sin \varphi_{1} \sin \Sigma, \\
& d_{31}=-\cos \varphi_{1} \sin \Sigma, d_{32}=\cos \varphi_{2} \sin \varphi_{1}-\cos \Sigma \cos \varphi_{1} \sin \varphi_{2}, \\
& d_{33}=\sin \varphi_{1} \sin \varphi_{2}+\cos \Sigma \cos \varphi_{1} \cos \varphi_{2}, d_{34}=V \sin \varphi_{1}+H_{2} \cos \varphi_{1} \sin \Sigma \\
& d_{41}=d_{42}=d_{43}=0, d_{44}=1
\end{aligned}
$$

\subsection{Pinion target tooth surface}

The contact path predesigned on the rotary projection of the gear real tooth surface has sole corresponding contact path on gear real tooth surface, pinion conjugate tooth surface $\Sigma_{g}$ and auxiliary tooth surface $\Sigma_{t}$. To obtain the pinion tooth surface that satisfy the predesigned $\mathrm{CP}$ and in point contact with gear real tooth surface at every contact position. Firstly, the contact path NMP on pinion auxiliary tooth surface(shown in Fig.8) is worked out according to the relationship of position vectors at contact point. $\Delta \varphi_{2}\left(\varphi_{1 N}\right), \Delta \varphi_{2}\left(\varphi_{1 M}\right), \Delta \varphi_{2}\left(\varphi_{1 P}\right)$ are TEs corresponding to meshing points $N, M$ and $P, \Delta \varphi_{2}\left(\varphi_{1 M}\right)=0$, the reasonable selection of the angles $\varphi_{1 N}$ and $\varphi_{1 P}$ can effectively avoid the jump phenomenon of TE curves during meshing, and consequently avoid the impact at meshing in and meshing out position.

Pinion auxiliary tooth surface $\Sigma_{t}$ is modified with a three-segment parabolic curve along the contact lines at each contact position to control the contact area. The 
peak of the modification curve is on the curve NMP, as shown in Fig.9. The modification along the contact line is carried out with parabola I inside the designed contact area EFGH, however, in the outside(AEHD and FBCG), the modification is carried out with parabola II(Fig.10), that means more material is removed outside the contact area. Thus the pinion target tooth surface $\Sigma_{1}$ is obtained.

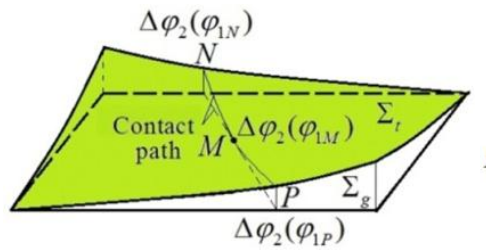

Fig.8 Auxiliary tooth surface

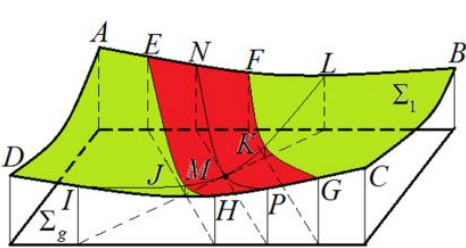

Fig.9 Target tooth surface

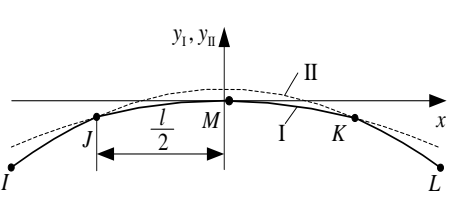

Fig.10 Modification curves

The equations of parabola I and II are as follows:

$$
\left\{\begin{array}{l}
y_{\mathrm{I}}=-\frac{\delta}{a^{2}} x^{2} \\
y_{\mathrm{II}}=-\left(\frac{\delta}{a^{2}}+a^{\prime}\right) x^{2}+Y
\end{array}\right.
$$

Where, $a$ is half the length of the major axis of the instant contact ellipse, $\delta$ is the elastic deformation, $Y=a^{\prime} l^{2} / 4$ represents the distance between two peaks of parabola, $l=2 a, \quad a^{\prime}$ is used to control the modification outside the predesigned contact area. The modification along the contact line is then denoted as $\delta_{y}$ :

$$
\delta_{y}=\left\{\begin{array}{l}
y_{\mathrm{I}}=-\frac{\delta}{a^{2}} x^{2},-\frac{l}{2} \leq x \leq \frac{l}{2} \\
y_{\mathrm{II}}=-\left(\frac{\delta}{a^{2}}+a^{\prime}\right) x^{2}+Y, x<-\frac{l}{2} \text { or } x>\frac{l}{2}
\end{array}\right.
$$

According to the distribution of pinion measuring points, the pinion auxiliary tooth surface $\Sigma_{t}$, target tooth surface $\Sigma_{1}$ and real tooth surface are then divided into $q(q=15 \times 15)$ mesh points, thus the position and unit normal vectors of mesh point $l(l=1,2, \ldots, q)$ of pinion target tooth surface are denoted as $\boldsymbol{r}_{T 1}{ }^{(l)}$ and $\boldsymbol{n}_{T 1}{ }^{(l)}$ : 


$$
\left\{\begin{aligned}
\boldsymbol{r}_{T 1}{ }^{(l)}\left(s, f, \varphi_{1}\right)= & \boldsymbol{r}_{A 1}{ }^{\left({ }^{()}\right.}+\delta_{y}{ }^{(l)} \boldsymbol{n}_{A 1}{ }^{\left({ }^{(l)}\right.} \\
\boldsymbol{n}_{T 1}{ }^{(l)}\left(s, f, \varphi_{1}\right)= & \frac{\frac{\partial \boldsymbol{r}_{T 1}{ }^{(l)}}{\partial_{s}} \times \frac{\partial \boldsymbol{r}_{T 1}{ }^{(l)}}{\partial_{f}}}{\left|\frac{\partial \boldsymbol{r}_{T 1}{ }^{(l)}}{\partial_{s}} \times \frac{\partial \boldsymbol{r}_{T 1}{ }^{(l)}}{\partial_{f}}\right|}
\end{aligned}\right.
$$

Then the normal deviation $\delta_{T M}{ }^{(l)}$ between pinion real and target tooth surface at point $l$ is obtained by the following equation:

$$
\boldsymbol{\delta}_{T M}{ }^{(l)}\left(s, f, \varphi_{1}\right)=\left(\boldsymbol{r}_{T 1}{ }^{(l)}-\boldsymbol{r}_{M 1}{ }^{(l)}\right) \cdot \boldsymbol{n}_{M 1}{ }^{(l)}
$$

\section{Adjustments of pinion machining parameters}

To make the pinion corrected tooth surface close to the target tooth surface, the machining parameters of pinion need to be adjusted. Since the predesigned TE curve is a second-order parabola, the following third-order roll ratio function is introduced:

$$
\phi_{c 2}\left(\phi_{1}\right)=\frac{1}{R_{a}} \phi_{1}+C_{1} \phi_{1}^{2}+C_{2} \phi_{1}^{3}
$$

Finally, an optimization model is created to solve the adjustments of pinion machining parameters, see Eq.(19). This optimization model sets the adjustments of pinion machining parameters $\Delta \zeta\left(\zeta\right.$ and $C_{1}, C_{2}$, not including $\left.R_{a}\right)$ as variables and the least sum of square errors of two sides as object, meanwhile the weight factor $w$ is introduced to selectively control the meshing performance of a specific side, because the adjustments of the machine setting parameters have synchronous impacts on both sides.SQP is used here as a tool to solve this model. 


$$
\left\{\begin{array}{l}
\min f(\Delta \zeta)=w f_{X}+(1-w) f_{V} \\
f_{X}=\sum_{k=1}^{q}\left(\delta_{X}^{(k)}\right)^{2} \\
f_{V}=\sum_{k=1}^{q}\left(\delta_{V}^{(k)}\right)^{2} \\
\Delta \zeta \in\left[\chi_{1}, \chi_{2}\right], \quad w \in[0,1]
\end{array}\right.
$$

Where, the subscript $X$ and $V$ represent the convex and the concave side. $\chi_{1}$ and $\chi_{2}$ represent the bottom and top limitations of adjustments of all the machining parameters, $[-1,1]$ is chosen for every machining parameter in this work.

\section{Numerical example}

This numerical example applies the proposed active tooth surface design methodology to modify a Klingelnberg-Oerlikon's Spirac high speed axle hypoid gear set cut by $\mathrm{C} 28$ hypoid generator. The basic parameters, machine setting parameters, cutter parameters of the gear pair are shown in Table 1, Table 2 and Table 3.

\section{Table 1}

Basic parameters

\begin{tabular}{|c|c|c|}
\hline & Gear(RH) & Pinion(LH) \\
\hline Shaft angle(deg) & & \\
\hline Offset(mm) & & \\
\hline Mean normal module ( $\mathrm{mm})$ & & \\
\hline Axial setting(mm) & 2.146 & -0.875 \\
\hline Number of teeth & 39 & 9 \\
\hline Face width(mm) & 28 & 31.53 \\
\hline Pitch cone angle(deg) & 72.026 & 17.325 \\
\hline Mean spiral angle(deg) & 49.997 & 34.046 \\
\hline Mean $\operatorname{radius}(\mathrm{mm})$ & 76.5 & 22.756 \\
\hline
\end{tabular}

Table 2

Machine setting parameters

\begin{tabular}{lcc} 
& Gear & Pinion \\
\hline Tilt angle(deg) & 0 & 20.787 \\
Swivel angle(deg) & 0 & -162.669 \\
Radial setting(mm) & 101.196 & 100.468
\end{tabular}




\begin{tabular}{|c|c|c|c|c|c|}
\hline Initial cradle angle setting(deg) & \multicolumn{2}{|c|}{-56.541} & \multicolumn{3}{|c|}{74.368} \\
\hline Vertical offset(mm) & \multicolumn{2}{|c|}{0} & \multicolumn{3}{|c|}{21.709} \\
\hline Increment of machine center to back $(\mathrm{mm})$ & \multicolumn{2}{|c|}{0.0024} & \multicolumn{3}{|c|}{0.903} \\
\hline Sliding base feed setting $(\mathrm{mm})$ & \multicolumn{2}{|c|}{0} & \multicolumn{3}{|c|}{9.182} \\
\hline Machine root angle(deg) & \multicolumn{2}{|c|}{72.026} & \multicolumn{3}{|c|}{-0.531} \\
\hline Roll ratio & \multicolumn{2}{|c|}{-} & \multicolumn{3}{|c|}{4.291} \\
\hline \multicolumn{6}{|l|}{$\begin{array}{l}\text { Table } 3 \\
\text { Cutter parameters }\end{array}$} \\
\hline & \multicolumn{2}{|c|}{ Gear } & \multicolumn{3}{|c|}{ Pinion } \\
\hline & Concave & Convex & & Concave & Convex \\
\hline Number of cutter starts & & & 17 & & \\
\hline Cutter radius(mm) & 88.024 & 87.578 & & 87.697 & 87.951 \\
\hline Profile angle(deg) & -23.61 & 17.516 & & -23.007 & 18.059 \\
\hline Offset angle(deg) & 19.822 & 19.927 & & -19.839 & -19.899 \\
\hline Blade curvature radius(mm) & 443.88 & 445.93 & & 451.35 & 452.65 \\
\hline
\end{tabular}

The theoretical tooth surfaces of gear and pinion are shown in Fig 11. C28 hypoid generator(Fig.12) and P65 measuring center(Fig.13) are adopted to cut and measure the tooth surfaces. The final products and one pair of the measuring results are shown in Fig.14 and Fig.15. The deviations of gear and pinion tooth thickness at the middle point are $29 \mu \mathrm{m}$ and $-13 \mu \mathrm{m}$, respectively.

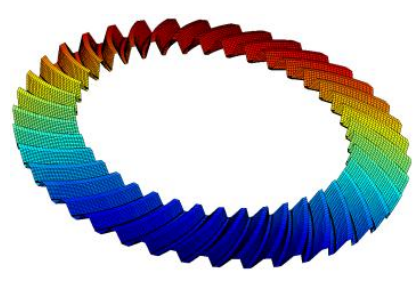

(a) Gear

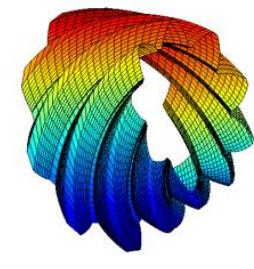

(b) Pinion

Fig.11 Theoretical tooth surfaces of gear and pinion

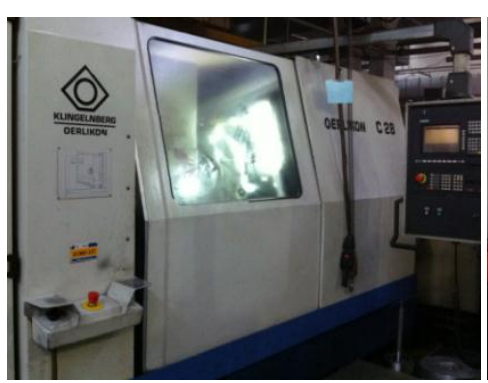

Fig.12 Practical cutting

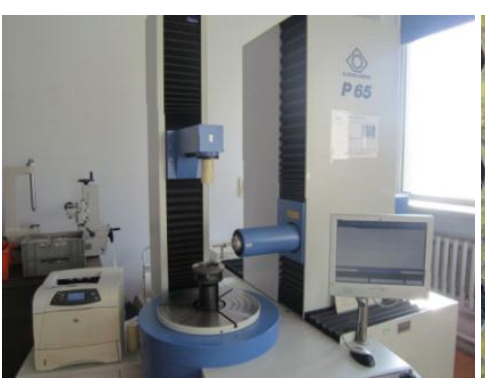

Fig.13 Gear under measuring

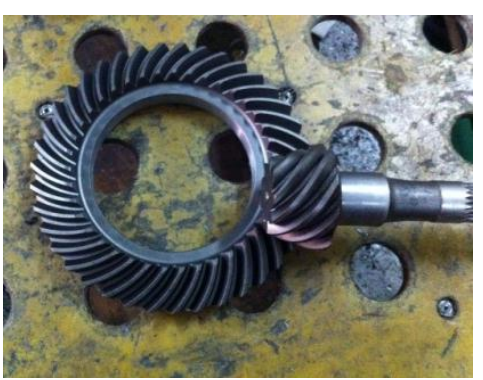

Fig.14 Final products 


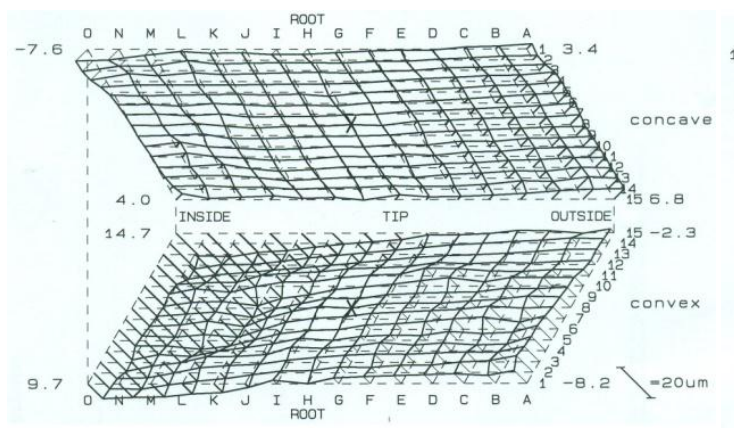

(a) Gear

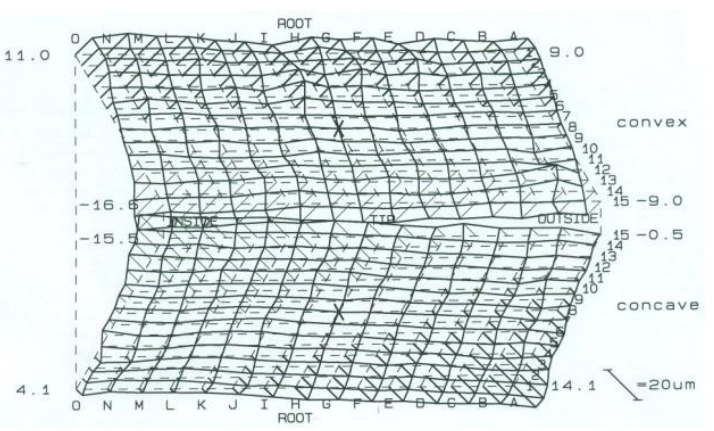

(b) Pinion

Fig.15 Deviations of tooth surfaces

According to the above-mentioned fitting method, the equations of the real tooth surfaces can be obtained as well as the second-order continuous bicubic NURBS surface and its control mesh of the drive side(concave side of pinion and convex side of gear) by fitting the discrete measuring coordinates, as shown in Fig.16 and Fig.17. The coast side can be obtained in the same way.

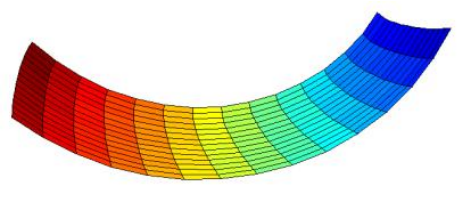

(a) NURBS surface

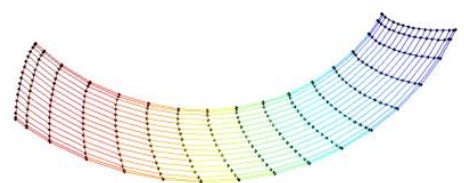

(b) control mesh

Fig. 16 Concave side of the pinion

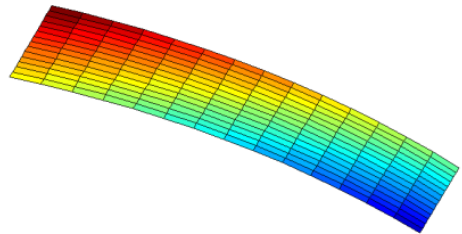

(a) NURBS surface

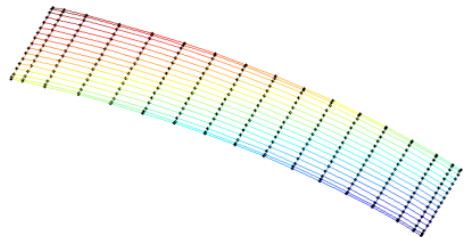

(b) control mesh

Fig. 17 Convex side of the gear

The preset parameters for the TE and CP are shown in Table 4. Finally, the pinion target tooth surface can be obtained, as shown in Fig.18.

Table 4

Preset parameters 


\begin{tabular}{lcc}
\hline$\delta_{\mathrm{TE}}(\operatorname{arc~sec})$ & 18 & 20 \\
$\eta(\operatorname{deg})$ & 140 & 40 \\
$a(\mathrm{~mm})$ & 4 & 4 \\
$a^{\prime}$ & 0 & 0.1 \\
\hline
\end{tabular}

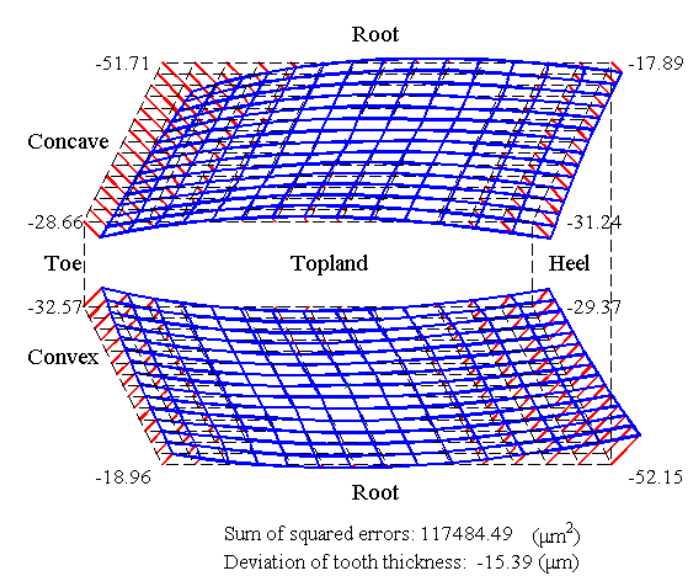

Fig.18 Deviations between pinion real and target tooth surface

When $w$ equals to $0.8,0.5$ and 0.2 , the adjustments of $\Delta \zeta$ are obtained via solving the Eq.(19),as shown in Table 5(only list $w=0.5$ ). The normal deviations between pinion corrected and target tooth surface are shown in Fig.19. The results comparison of different weight factors is shown in Table 6.

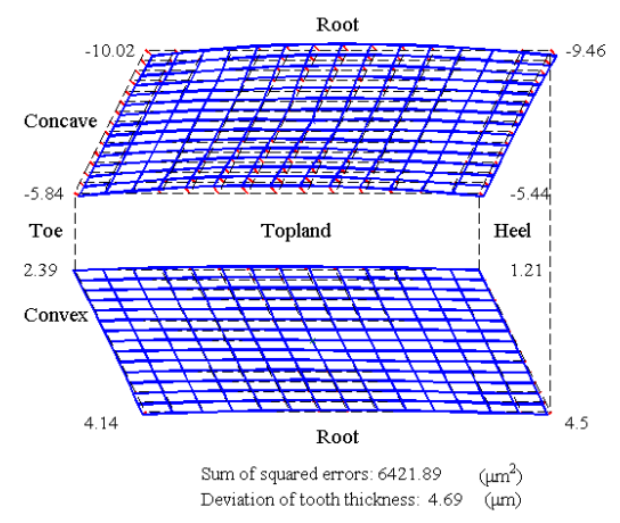

(a) $\quad w=0.8$

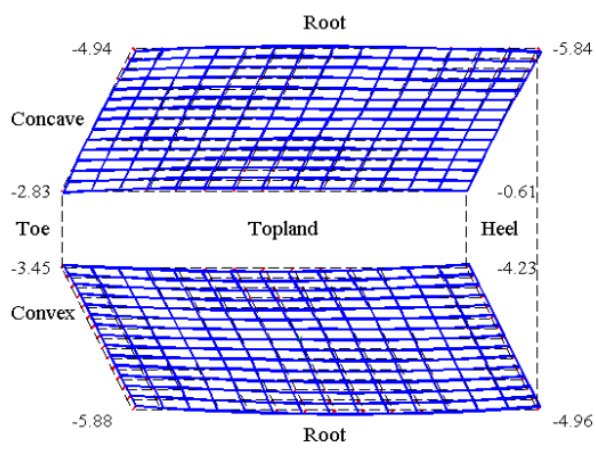

Sum of squared errors: $3812.68 \quad\left(\mu \mathrm{m}^{2}\right)$ Deviation of tooth thickness: $-2.36(\mu \mathrm{m})$

(b) $w=0.5$ 


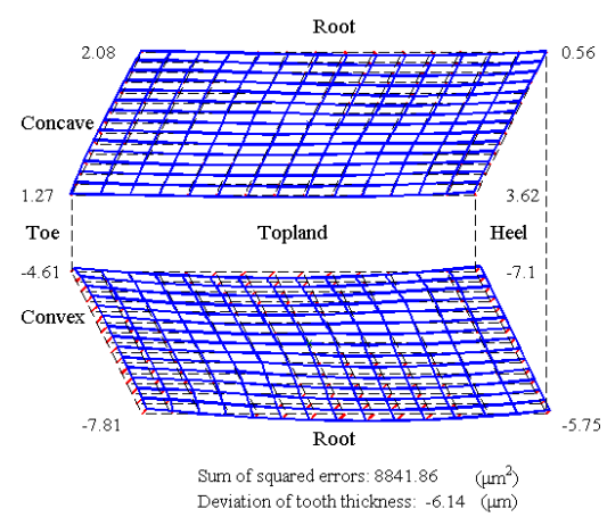

(c) $w=0.2$

Fig.19 Deviations between pinion modified and target tooth surface

Table 5

Modifications of pinion machining parameters

\begin{tabular}{|c|c|c|}
\hline & Concave & Convex \\
\hline Mean $\operatorname{radius}(\mathrm{mm})$ & 0.0436 & 0.0428 \\
\hline Profile angle(deg) & 0.0521 & 0.0537 \\
\hline Offset angle(deg) & - & - \\
\hline Blade curvature radius $(\mathrm{mm})$ & -0.0004 & -0.0002 \\
\hline Tilt angle(deg) & & \\
\hline Swivel angle(deg) & & \\
\hline Radial setting(mm) & & \\
\hline Initial cradle angle setting(deg) & & \\
\hline Vertical offset(mm) & & \\
\hline Increment of machine center to back $(\mathrm{mm})$ & & \\
\hline Sliding base feed setting $(\mathrm{mm})$ & & \\
\hline Machine root angle(deg) & & \\
\hline Modified roll motion coefficient $\mathrm{C} 1$ & & \\
\hline Modified roll motion coefficient $\mathrm{C} 2$ & & \\
\hline
\end{tabular}

\section{Table 6}

Results comparison of different weight factors

\begin{tabular}{lccc}
\hline & $\begin{array}{c}\text { Sum of squared } \\
\text { errors }\left(\mu \mathrm{m}^{2}\right)\end{array}$ & Maximum error $(\mu \mathrm{m})$ \\
\hline Initial & 117484.49 & -52.15 & concave \\
$w=0.8$ & 6421.89 & 4.5 & -51.71 \\
$w=0.5$ & 3812.68 & -5.88 & -10.02 \\
$w=0.2$ & 8841.86 & -7.81 & -5.84 \\
\hline
\end{tabular}

When $w=0.5$, as shown in Table 6 , the quadratic sum of deviations of two sides is the smallest. As $w$ increases, the absolute value of maximum deviation of 
convex side decreases while the concave side increases, and vice versa. That is, by adjusting the weight factor, the approaching level at a certain side can be selectively guaranteed, which means the meshing performances at this side will be more closer to the predesigned performances.

Take the case as $w=0.5$ for example, the meshing performances of pinion modified tooth surface and gear real tooth surface is analyzed. TCA results are shown in Fig.20, where the dashed line represents the predesigned contact line. The deviations between the real and the predesigned contact path are shown in Fig.21. Counting positively what goes to the heel direction. The series numbers of meshing points are arranged according to the order from meshing in to meshing out. The maximum deviations of the drive and coast sides are $0.22 \mathrm{~mm}$ and $0.14 \mathrm{~mm}$, respectively. The maximum TEs of the drive and coast sides are -18.8 arc sec and -21.3 arc sec, respectively, and their percentage deviations are only $4.44 \%$ and $6.5 \%$ compared to the predesigned values.

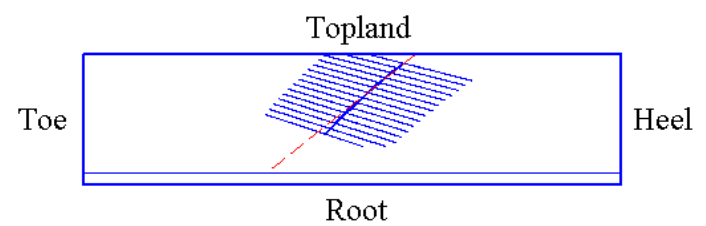

(a)Contact pattern for gear convex

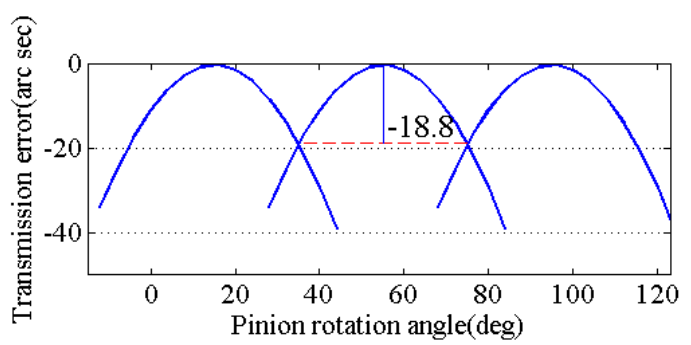

(c)Transmission error for drive side

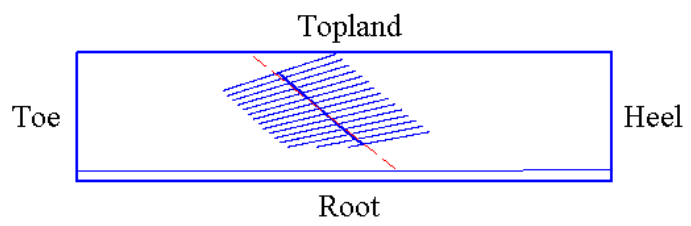

(b) Contact pattern for gear concave

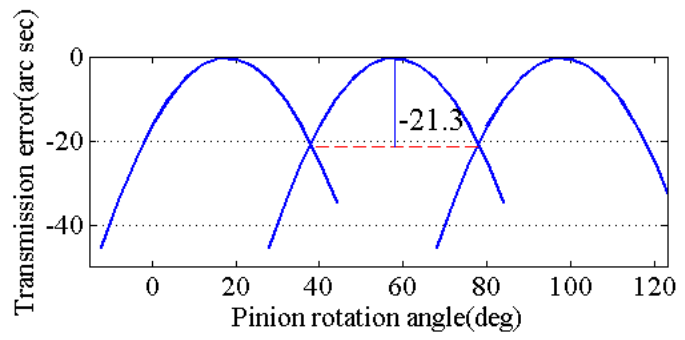

(d) Transmission error for coast side

Fig.20 TCA results 


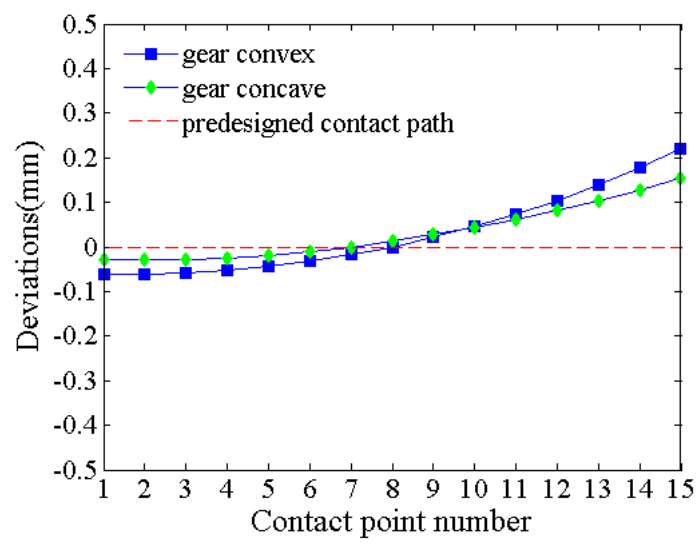

Fig.21 Deviations of contact paths

Rolling test results are shown in Fig.22. The results of the numerical example and the rolling test reveal that the predesigned meshing performance can be obtained by the above active tooth surface design methodology.

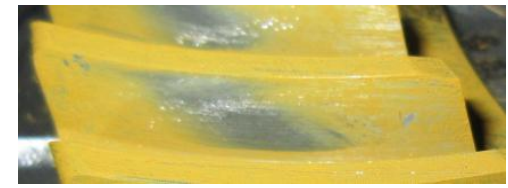

(a) Gear convex

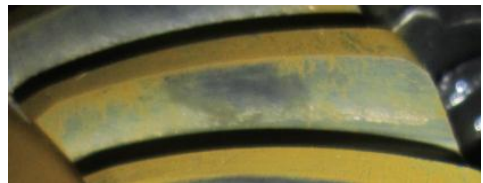

(b) Gear concave

Fig.22 Rolling test results

\section{Conclusions}

This paper proposes a new active tooth surface design methodology of face-hobbed hypoid gears with predesigned second-order function of TE and CP. It can be used in the phase of trial-manufacture and only one time of correction of the pinion real tooth surface is needed to get the desired meshing performances, but the errors of both the gear and pinion real tooth surfaces caused by machining errors and the deformation of heat treatment are all considered, and a weight factor is introduced to selectively guarantee the meshing performances of a certain side.

As the numerical example and rolling test clearly illustrates, the TCA results 
satisfies the predesigned meshing performances, with an error of only $4.44 \%$ and $6.5 \%$ in TE for drive and coast side, respectively, and the maximum deviations of contact path are $0.22 \mathrm{~mm}$ and $0.14 \mathrm{~mm}$ for two sides. This proposed active tooth surface design methodology allows the designers to design the tooth surfaces according to the measuring results and the meshing performances requirements, which is also beneficial to shorten the production cycle and can be applied to the other gear types in double-flank cutting.

\section{Acknowledgments}

The authors would like to thank the financial aid and support from the National Science Foundation of China (No. 51175423,51375384). We also thank anonymous reviewers and editors for their valuable comments and suggestions.

\section{References}

[1] F.L. Litvin, Y. Zhang, Local synthesis and tooth contact analysis of face-milled spiral bevel gears, NASA CR4342, Chicago: NASA Lewis Research Center, 1991.

[2] A. Fuentes, F.L. Litvin, B.R. Mullins, R. Woods, R.F. Handschuh, Design and stress analysis of low-noise adjusted bearing contact spiral bevel gears, ASME Journal of Mechanical Design 124(2002) 524-532.

[3] F.L. Litvin, A. Fuentes, Gear Geometry and Applied Theory, Seconded, Cambridge University Press, New York, 2004.

[4] X. Cao, Z. Fang, J. Zhang, Function-oriented active tooth surface design of spiral bevel gears[J].Chin. Journal of mechanical engineering.43(8)(2007)155-158.

[5] X. Cao, Z. Fang, H. Xu, Design of pinion machine tool-settings for spiral bevel gear by controlling contact path and transmission error [J], Chin. J. Aeronaut. 21(2)(2008) 179-186.

[6] I. Gonzalez-Perez, A. Fuentes, F.L. Litvin, et al., Modified surface topology of involute helical gears developed for improvement of bearing contact and reduction of transmission errors[C]//ASME, International Design Engineering Technical Conferences and Computers and Information in Engineering Conference, Am. Soc. Mech. Eng. 2005 (2005) 571-584.

[7] V. Simon, Optimal machine tool setting for hypoid gears improving load distribution, ASME Journal of Mechanical Design 123 (2001) 577-582. 
[8] P.Y. Wang, Z.H. Fong, Fourth-order kinematic synthesis for face-milling spiral bevel gears with modified radial motion (MRM) correction [J], J. Mech. Des. 128 (2) (2006) 457-467.

[9] C.K. Lee, Manufacturing process for a cylindrical crown gear drive with a controllable fourth order polynomial function of transmission error [J], J. Mater. Process. Technol. 209 (1) (2009) 3-13.

[10] J. Su, Z. Fang, X. Cai, Design and analysis of spiral bevel gears with seventh-order function of transmission error [J], Chin. J. Aeronaut. 26 (5) (2013) 1310-1316.

[11] J. Jiang, Z. Fang, Design and analysis of modified cylindrical gears with a higher-order transmission error, Mech. Mach.Theory. 88 (2015) 141-152

[12] A. Artoni, M. Kolivand, A. Kahraman, An ease-off based optimization of the loaded transmission error of hypoid gears [J], J. Mech. Des. 132 (2010) 011010.

[13] A. Artoni, M. Gabiccini, M. Guiggiani, A. Kahraman, Multi-objective ease-off optimization of hypoid gears for their efficiency,noise,and durability performacnes[J], J. Mech. Des. 133 (2011) 121007.

[14] Y.P. Shih, A novel ease-off flank modification methodology for spiral bevel and hypoid gear, Mech. Mach.Theory. 45(8)(2010)1108-1124.

[15] Kawasaki. K, Tsuji. I, Gunbara. H, Houjoh. H. Method for remanufacturing large-sized skew bevel gears using CNC machining center, Mech. Mach.Theory.92 (2015) 213-229.

[16] X. Dong, Design and manufacture for epicycloidal spiral bevel and Hypoid Gears, China Machine Press, Beijing,2002.

[17] Y.P. Shih, Z.H. Fong, Mathematical model for the universal face-hobbing hypoid gear generator[J], J. Mech. Des. 128 (2007) 062604.

[18] X. Zhu, Free surface modeling technique, China Science Press, Beijing,2001.

[19] J. Du, Z. Fang, H. Gao, Y. Zhang, G. Zhao, Contact analysis of cycloid tooth hypoid gear based on real tooth surface [J]. Journal of south china university of technology (natural science edition), 42 (3)( 2015) 35-40. 


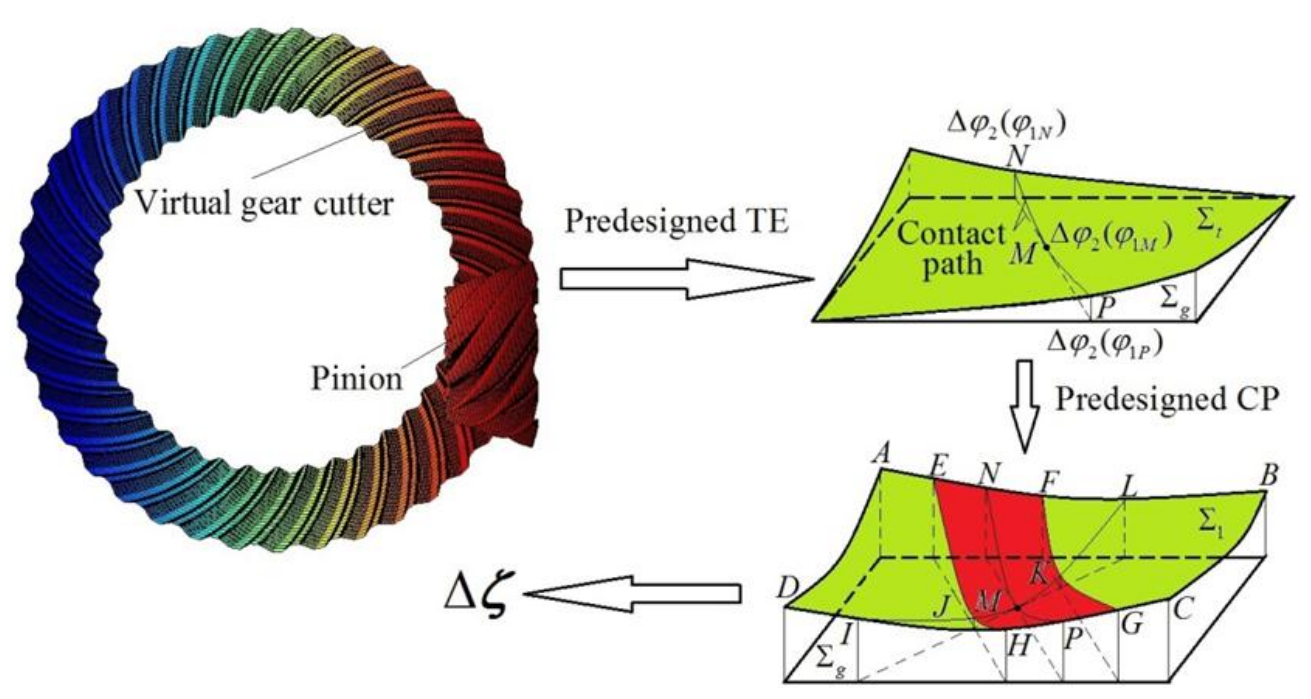

\section{Graphical Abstract}

As the Fig shows, Firstly, an auxiliary tooth surface of pinion $\Sigma_{t}$ is generated by using the gear real tooth surface as a virtual cutter, $\Sigma_{t}$ is in line contact with gear real tooth surface under a predesigned transmission error (TE), then the target tooth surface of pinion $\Sigma_{1}$ is obtained by modifying $\Sigma_{t}$ along the contact lines according to the predesigned contact pattern $(\mathrm{CP})$, thus the predesigned meshing performances are satisfied. Finally, the adjustments of pinion machining parameters are solved via an optimization model. 